\title{
A Bayesian Network under Strict Chain Model for Computing Flow Risks in Smart City
}

\author{
Zengfanxiang Wei $\mathbb{D}^{1}$ Lei Zhang $\mathbb{D}^{1},{ }^{1}$ Qi Yue $\mathbb{D}^{2},{ }^{2}$ and Muchen Zhong $\mathbb{D}^{3}$ \\ ${ }^{1}$ School of Economics and Management, Beijing Jiaotong University, Beijing 100044, China \\ ${ }^{2}$ School of Information Management, Jiangxi University of Finance and Economics, Nanchang 330013, China \\ ${ }^{3}$ Department of Cyber Security, Lancaster University, Lancaster LA2 OPF, Lancaster, UK
}

Correspondence should be addressed to Lei Zhang; 19125341@bjtu.edu.cn

Received 5 April 2020; Accepted 19 May 2020; Published 8 June 2020

Guest Editor: Zhihan Lv

Copyright (c) 2020 Zengfanxiang Wei et al. This is an open access article distributed under the Creative Commons Attribution License, which permits unrestricted use, distribution, and reproduction in any medium, provided the original work is properly cited.

\begin{abstract}
Risk management is a key factor for smart city running. There are many risk events in a strict process like transportation management of a smart city or a medical surgery in a smart hospital, and every step may lead to one kind of risk or more. In view of the fact that the occurrence of the flow risks follows the sequence formed by each process step, this paper presents a Bayesian network under strict chain (BN_SC) to model this situation. In this model, the probabilistic reasoning formula is given according to the sequence of process steps, and the probabilities given by the model can do risk factor analysis to support the system to find an effective way to improve the process like machine manufacturing or a medical surgery. Finally, an example is analyzed based on the information given by doctors according to the situation of LC in their hospital located in Sichuan Province of China, which shows the effectiveness and rationality of the proposed BN_SC model.
\end{abstract}

\section{Introduction}

Machine learning has applications in various fields for smart city running, such as machinery manufacturing, especially in probability evaluation [1-3]. Bayesian learning as a machine learning method can estimate the probabilities of risk occurrence like medical risk events in the medical process [4-7]. One or more of which will lead to the occurrence of other risk events, for example, in laparoscopic cholecystectomy, trocar puncture may cause intestinal injury, which often leads to infection.

In this kind of situation, the correlations of events can be quantified in a Bayesian network $[8,9]$. However, the occurrence of risk events connected with the medical process, for example, LC must follow the surgical steps that construct a strict chain for the Bayesian net to follow. Existing quantitative research rarely studies on models for this special feature. According to this situation, we present a Bayesian network under strict chain (BN_SC) model for the first time. In this model, the probabilistic reasoning formula according to the sequence of surgical steps processes the logical inference for the probability of each risk subjected to the strict chain constructed by the surgical steps of LC. BN_SC model can calculate the probabilities of risk flow more effectively than the traditional Bayesian network.

The remainder of the paper is organized as follows. Section 2 will review the research about the Bayesian network and we will illustrate the process of LC with a directed acyclic graph in Section 3. In Section 4, the BN_SC model will be proposed, including the logical inference of the risks events and the formula to compute the probabilities of risks in LC. Finally, an example is analyzed based on the information given by doctors according to the situation of laparoscopic cholecystectomy in their hospital, which shows the effectiveness and rationality of the BN_SC in Section 5.

\section{Related Works}

Previous studies have comprehensively expounded the concept and related properties of Bayesian networks. Tuyls and Maes pointed out that a Bayesian network is a directed acyclic graph, which consists of a set of random variables. 
The meaning from node $X$ to node $Y$ is that node $X$ has a direct influence on node $Y$ [10]. The main feature of the Bayesian network is that it shows the interdependent relationship between variables. Koller and Friedman et al., Meinshausen and Btihlmann, and Wainwright and Jordan pointed out that the graph connected by undirected edges between variables is an undirected graph known as Markov network [11-13]. These graphs produce undirected graph models, which are more suitable for analyzing the similarity and related behavior between variables. Ghahramani, Heckerman et al., and Neapolitan pointed out that a graph connected by a directed edge of variables is a "directed graph," and a directed graph without a cycle is a "directed acyclic graph" (DAG) [14-16]. Directed acyclic graphs are usually based on the concept of family order. For example, in $A \longrightarrow B \longrightarrow C, A$ is the parent node of $B, C$ is the child node of $B, A$ and $B$ are the ancestors of $C$, and $B$ and $C$ are the descendants of $A$. This ranking clearly expresses causality, and these figures produce a class of models called Bayesian networks. Andersson et al., Gillispie and Perlman, and Pearl have pointed out that some directed acyclic graphs have twoway edges, which are suitable for situations where some variables interact with each other [17-19]. They represent a class of Markov equivalent directed acyclic graphs.

Bayesian network is widely used in risk evaluation research. Ronald et al. show that chains of events can lead to increased or critical emissions of volatile organic compounds by assessing risks based on the Bayesian network [20]. Bhattacharjee applied a Bayesian model that can handle death data analysis without stratifying in the presence of competing risks [21]. Sanchez et al. develop a general framework and a method to estimate the impact of project management maturity on project performance by using Bayesian networks to formalize project management experts' knowledge [22]. Masmoudi et al. used a discrete Bayesian network with a latent variable to model the payment default of loan subscribers [23]. Punyamurthula and Badurdeen represented operational risks of production line and their causal relationships based on Bayesian Belief Networks [24].

\section{Process of $\mathrm{LC}$}

Laparoscopic surgery is a new method of minimally invasive surgery, which is the inevitable trend of the future development of surgical methods. Cholecystectomy is one of the most common abdominal operations, which is usually performed in laparoscopic surgery in developed countries. For example, $90 \%$ of cholecystectomy in the United States is performed by laparoscopy. Laparoscopic cholecystectomy is regarded as the "gold standard" for surgical treatment of cholelithiasis $[25,26]$.

Laparoscopic cholecystectomy is divided into five steps, which include trocar puncture (into the abdomen), separation of adhesion, anatomy bile triangle, dissection of gallbladder bed, and removal and suture of gallbladder. In the surgery, intestinal injury may be caused by trocar puncture, bile duct injury may be caused by separation of adhesion, anatomy of bile triangle, and dissection of gallbladder, and vascular injury may be caused by removal and suture of gallbladder. Moreover, the three kinds of injuries may lead to infection and massive hemorrhage, which can eventually lead to death. In order to describe the risk probability in LC surgery more succinctly, Table 1 shows the mathematical symbols in this paper.

According to analysis, we can get the directed acyclic graph of LC operation as shown in Figure 1.

Figure 1 describes the specific process how the LC works. We can build a special model by following the logic derived from the strict chain. As shown in the figure, the risk of $R_{2}$ happened in the process of Step 2. Moreover, the occurrence of $R_{1}$ means the failure of Step 1, which means the surgery cannot be performed and $R_{2}$ cannot occur. The five surgery steps formed a chain above the Bayesian network of risks in the surgery. The occurrence of each risk needs to follow the order of the steps in the chain strictly. This changes the rule of the traditional inference of Bayesian network. To solve the problem, we present a new model in Section 4.

\section{Bayesian Network under Strict Chain (BN_SC)}

According to the analysis in Section 2, we build a Bayesian network to model the LC process. Different from the traditional Bayesian network, in many medical procedures, the risk Bayesian network is developed under the surgical process such as the Bayesian network of LC surgery in Section 2, and the surgical process is characterized by the fact that the next step can only be taken after the previous step surgical procedure is successful. So those medical steps construct a strict process chain naturally such as $S_{1}$ to $S_{5}$ of LC, and more importantly, the Bayesian network of surgery risks should follow the steps of the chain model. According to this characteristic, this paper proposes Bayesian network under strict chain model. Under BN_SC model, if $j=i+1$, then surgical step $j$ can only begin after surgical Step $i$ completes successfully. The formula for calculating the probability of the risk occurrence of the event $j$ is as follows:

$$
P\left(S_{j}\right)=\prod_{i=1}^{j-1} P\left(S_{1}\right) P\left(S_{i}\right) .
$$

Here, $P\left(D_{i}\right)$ means the probability of the surgical Step $i$ completed successfully. This paper assumed that only when the surgical Step $i$ causes the risk $i$ will lead to the unsuccessful surgery, and some other unexpected factors such as power outages are not under consideration. So, $P\left(D_{i}\right)$ and $P\left(R_{j}\right)$ form complementary relationship, that is, $P\left(R_{j}\right)=1-P\left(D_{i}\right)$. In laparoscopic surgery, the patient is bound to have the first surgical step after entering the procedure, so the first surgical step is bound to occur here, that is, $P\left(S_{1}\right)=1$.

Therefore, the probability of intestinal injury risk $P\left(R_{1}\right)$ caused by the first surgical procedure can be represented as follows:

$$
P\left(R_{1}\right)=P\left(R_{1} \mid S_{1}\right) P\left(S_{1}\right)=P\left(R_{1} \mid S_{1}\right) .
$$


TABLE 1: List of symbol notations and description.

\begin{tabular}{lc}
\hline Symbols & Descriptions \\
\hline$S_{1}$ & Trocar puncture (into the abdomen) happened \\
$S_{2}$ & Separation of adhesion happened \\
$S_{3}$ & Anatomy of bile triangle happened \\
$S_{4}$ & Dissection of gallbladder bed happened \\
$S_{5}$ & Removal and suture of gallbladder happened \\
$D_{1}$ & Trocar puncture (into the abdomen) has done \\
$D_{2}$ & successfully \\
$D_{3}$ & Separation of adhesion has done successfully \\
$D_{4}$ & Anatomy of bile triangle has done successfully \\
$D_{5}$ & Dissection of gallbladder bed has done successfully \\
$R_{1}$ & Removal and suture of gallbladder has done \\
$R_{2}$ & successfully \\
$R_{3}$ & Intestinal injury \\
$R_{4}$ & Bile duct injury \\
$R_{5}$ & Vascular injury \\
$R_{6}$ & Infection \\
$W_{1}, W_{2}, W_{3}$ & Massive hemorrhage \\
$W_{4}, W_{5}, W_{6}$ & Death \\
& Contribution ratio of $R_{1}, R_{2}, R_{3}$ to $R_{4}$ \\
&
\end{tabular}

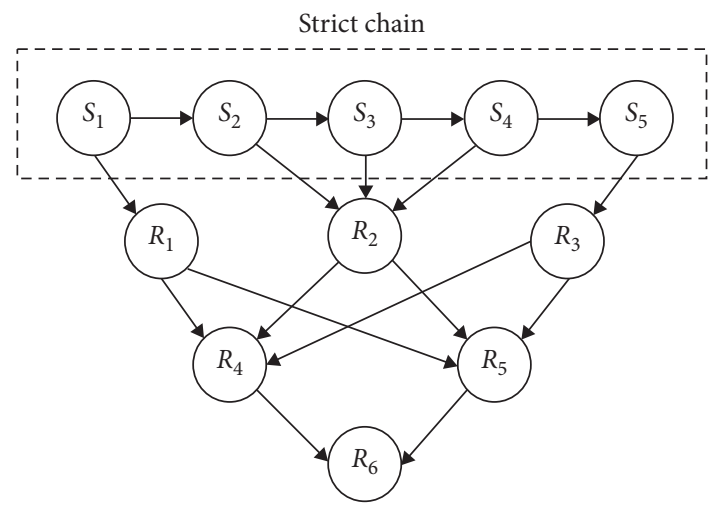

FIGURE 1: Bayesian network under a strict chain.

The probability of bile duct injury risk $P\left(R_{2}\right)$ caused by multiple surgical procedures $S_{2}, S_{3}$, and $S_{4}$ can be represented as follows:

$$
\begin{aligned}
P\left(R_{2}\right)= & P\left(R_{2} \mid S_{2}\right) P\left(S_{2}\right)+P\left(R_{2} \mid S_{3}\right) P\left(S_{2} \mid S_{2}\right) P\left(S_{2}\right)+ \\
& P\left(R_{2} \mid S_{4}\right) P\left(S_{4} \mid S_{3}\right) P\left(S_{3} \mid S_{2}\right) P\left(S_{2}\right) \\
= & \left(1-P\left(D_{2}\right)\right) P\left(D_{1}\right)+\left(1-P\left(D_{3}\right)\right) P\left(D_{2}\right) P\left(D_{1}\right)+ \\
& \left(1-P\left(D_{4}\right)\right) P\left(D_{3}\right) P\left(D_{2}\right) P\left(D_{1}\right) \\
= & P\left(D_{1}\right)\left(1-P\left(D_{2}\right) P\left(D_{3}\right) P\left(D_{4}\right)\right) .
\end{aligned}
$$

Moreover, the probability of incision tear risk $P\left(R_{3}\right)$ can be represented as follows:

$$
\begin{aligned}
P\left(R_{3}\right) & =P\left(R_{3} \mid S_{5}\right) P\left(S_{5}\right) \\
& =P\left(R_{3} \mid S_{5}\right) P\left(D_{4}\right) P\left(D_{3}\right) P\left(D_{2}\right) P\left(D_{1}\right) .
\end{aligned}
$$

According to the DAG of LC, three risks can lead to the risk of infection $R_{4}$ and the risk of massive hemorrhage $R_{5}$. However, this conditional probability cannot be calculated by statistics. It can only be quantitatively judged based on the experience accumulated by doctors in completed cases. In order to get this judgement value, two sets of comparison matrices are given by doctors who are allowed to score 1-9. The numbers 3, 5, 7, and 9 correspond to the verbal judgments "moderately more dominant," "strongly more dominant," "very strongly more dominant," and "extremely more dominant" (with 2, 4, 6, and 8 for compromise between the previous values). We are permitted to interpolate values between the integers, if desired, or use numbers from an actual ratio scale of measurement. This scale is mathematically derived from stimulus-response theory and has been extended through the use of structuring and decomposition to assume arbitrarily large values as necessary. Firstly, for risk $R_{4}$, we use pairwise comparison to research which risk $R_{1}, R_{2}, R_{3}$ is more likely to cause $R_{4}$. After entering scores given by doctors into matrix, the contribution ratio of three kinds of risk $R_{1}, R_{2}, R_{3}$ to $R_{4}$ is obtained by calculating the eigenvector, and the contribution ratio of $R_{1}, R_{2}, R_{3}$ can be represented as $w_{1}, w_{2}, w_{3}$ :

$$
\left.\begin{array}{l|l}
R_{4} & R_{1} R_{2} R_{3} \\
\hline R_{1} & \\
R_{2} & R_{i j} \\
R_{3}
\end{array}\right) \longrightarrow\left(\begin{array}{l}
w_{1} \\
w_{2} \\
w_{3}
\end{array}\right) .
$$

By the same token, the contribution ratio of $R_{1}, R_{2}, R_{3}$ to $R_{5}$ can be represented as $w_{4}, w_{5}, w_{6}$ :

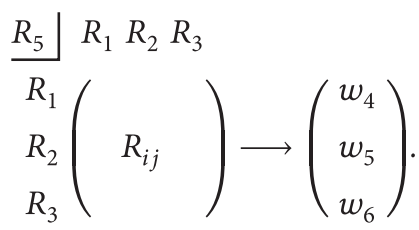

In addition, by comparing the risk of massive bleeding and the risk of infection leading to death, the weight of risk $R_{4}, R_{5}$ can be obtained, which can be represented by $C_{0}, C_{1}$ :

$$
\begin{array}{l|l}
R_{6} & R_{4} R_{5} \\
R_{4} & \left(\begin{array}{l}
R_{i j} \\
R_{5}
\end{array}\right) \longrightarrow\left(\begin{array}{l}
C_{0} \\
C_{1}
\end{array}\right) .
\end{array}
$$

Then, the final weight $C_{2}, C_{3}, C_{4}$ of $R_{1}, R_{2}, R_{3}$ can be obtained by the following formula:

$$
\begin{aligned}
& C_{2}=C_{0} w_{1}+C_{1} w_{4}, \\
& C_{3}=C_{0} w_{2}+C_{1} w_{5}, \\
& C_{4}=C_{0} w_{3}+C_{1} w_{6} .
\end{aligned}
$$

Based on the above formula, we can use $P\left(D_{1}\right), P\left(D_{2}\right), P\left(D_{3}\right), P\left(D_{4}\right), P\left(D_{5}\right)$ to express the probability of death. According to the following formula, we 
analyze the relations between each surgery step and death risk:

$$
\begin{aligned}
P\left(R_{6}\right)= & C_{0} P\left(R_{4}\right)+C_{1} P\left(R_{5}\right)=C_{2} P\left(R_{1}\right) \\
& +C_{3} P\left(R_{2}\right)+C_{4} P\left(R_{3}\right) \\
= & C_{2}\left(1-P\left(D_{1}\right)\right)+C_{3} P\left(D_{1}\right)\left(1-P\left(D_{2}\right) P\left(D_{3}\right) P\left(D_{4}\right)\right) \\
& +C_{4}\left(1-P\left(D_{5}\right)\right) P\left(D_{4}\right) P\left(D_{3}\right) P\left(D_{2}\right) P\left(D_{1}\right) \\
= & C_{2}+\left(C_{3}-C_{2}\right) P\left(D_{1}\right)+\left(C_{4}-C_{3}\right) P\left(D_{1}\right) P \\
& \cdot\left(D_{2}\right) P\left(D_{3}\right) P\left(D_{4}\right) \\
& -C_{4} P\left(D_{1}\right) P\left(D_{2}\right) P\left(D_{3}\right) P\left(D_{4}\right) P\left(D_{5}\right) .
\end{aligned}
$$

\section{Application: Validating Our BN_SC Model}

To further illustrate the calculation process of proposed method, we implement the BN_SC model to a hospital in Sichuan Province of China. According to the existing laparoscopic cholecystectomy records of surgical cases, the hospital counted 1166 cases in the past year. The statistical results demonstrate the success ratios of trocar puncture, separation of adhesion, anatomy of bile triangle, dissection of gallbladder bed, and removal and suture of gallbladder were $0.990,0.970,0.873,0.830$, and 0.821 . The probability of intestinal injury is 0.010 according to formula (2), and the probability of bile duct injury and vascular injury is 0.294 and 0.125 according to formula (3).

In addition, information about death, infection, and massive bleeding involves the privacy of patients, so doctors can only judge the relationship between them based on experience instead of providing specific values. According to the method given in Section 3, we asked the attending physician of laparoscopic cholecystectomy operation to give the subjective evaluation as follows:

Three surgical risk comparison matrices for infection are described as follows:

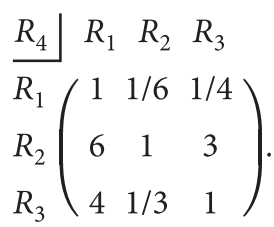

By finding the eigenvector of the matrix, the weight is obtained:

$$
\left(\begin{array}{l}
w_{1}=0.09 \\
w_{2}=0.64 \\
w_{3}=0.27
\end{array}\right) .
$$

Three surgical risk comparison matrices for massive hemorrhage are described as follows:

$$
\begin{aligned}
& \begin{array}{l|lll}
R_{5} & R_{1} & R_{2} & R_{3}
\end{array} \\
& \begin{array}{l}
R_{1} \\
R_{2}
\end{array}\left(\begin{array}{ccc}
1 & 3 & 1 / 5 \\
1 / 3 & 1 & 1 / 7 \\
5 & 7 & 1
\end{array}\right)
\end{aligned}
$$

By finding the eigenvector of the matrix, the weight is obtained:

$$
\left(\begin{array}{l}
w_{4}=0.19 \\
w_{5}=0.08 \\
w_{6}=0.73
\end{array}\right) .
$$

The surgical risk comparison matrices for death are described as follows:

$$
\begin{aligned}
& \begin{array}{l|ll}
R_{6} & R_{4} & R_{5}
\end{array} \\
& R_{4}\left(\begin{array}{cc}
1 & 1 / 2 \\
2 & 1
\end{array}\right) \text {. }
\end{aligned}
$$

By finding the eigenvector of the matrix, the weight is obtained:

$$
\begin{aligned}
& C_{0}=0.333, \\
& C_{1}=0.666 .
\end{aligned}
$$

We can obtain $C_{2}, C_{3}, C_{4}$ by formulas (8)-(10):

$$
\begin{aligned}
& C_{2}=0.157, \\
& C_{3}=0.267, \\
& C_{4}=0.576 .
\end{aligned}
$$

According to the analysis, the death risk of laparoscopic cholecystectomy surgery in a hospital in Sichuan can be obtained as follows:

$$
\begin{aligned}
P\left(R_{6}\right)= & C_{0} P\left(R_{4}\right)+C_{1} P\left(R_{5}\right)=C_{2} P\left(R_{1}\right)+C_{3} P\left(R_{2}\right)+C_{4} P\left(R_{3}\right) \\
= & C_{2}+\left(C_{3}-C_{2}\right) P\left(D_{1}\right)+\left(C_{4}-C_{3}\right) P\left(D_{1}\right) P \\
& \cdot\left(D_{2}\right) P\left(D_{3}\right) P\left(D_{4}\right) \\
& -C_{4} P\left(D_{1}\right) P\left(D_{2}\right) P\left(D_{3}\right) P\left(D_{4}\right) P\left(D_{5}\right) \\
= & 0.157 \times 0.010+0.267 \times 0.294+0.576 \times 0.125 \\
= & 0.152 .
\end{aligned}
$$

The BN_SC model is more effective than the traditional Bayesian model in reducing the computations of the particular conditional probabilities, because some of the conditional probabilities can be replaced by the probabilities of ex-step.

Sensitivity analysis for the quantitative relationships between the success rates of each laparoscopic cholecystectomy surgery step and the probability of death are as follows from Figure 2 to Figure 11:

$$
\begin{aligned}
& P\left(R_{6}\right)=0.157-0.005 P\left(D_{1}\right), \\
& P\left(R_{6}\right)=0.266-0.117 P\left(D_{2}\right), \\
& P\left(R_{6}\right)=0.266-0.130 P\left(D_{3}\right), \\
& P\left(R_{6}\right)=0.266-0.137 P\left(D_{4}\right), \\
& P\left(R_{6}\right)=0.215-0.077 P\left(D_{5}\right) .
\end{aligned}
$$

Through the above experimental results, it can be observed that the most effective way to reduce the risk of death is to improve the success rate of dissection of gallbladder, and the second is to improve the success rate of anatomical 


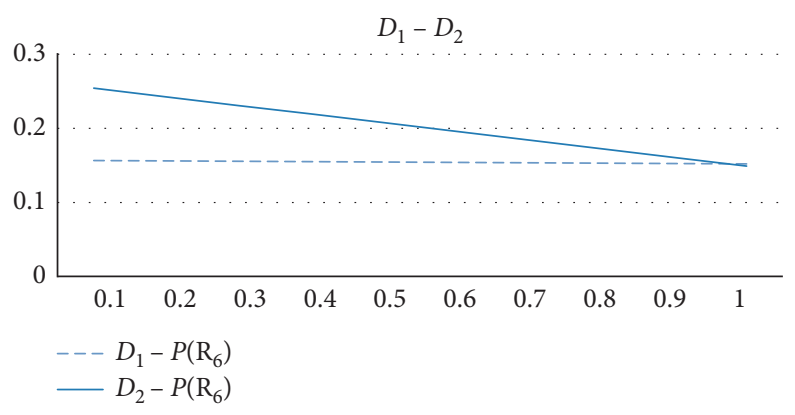

Figure 2: Sensitivity analysis of $D_{1}-D_{2}$.

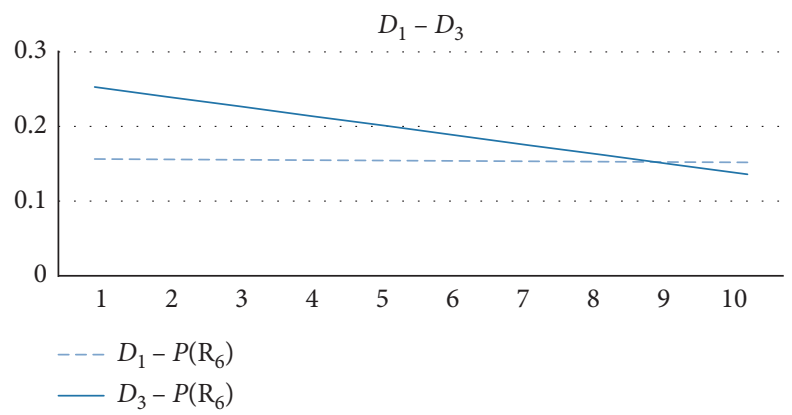

FIgURe 3: Sensitivity analysis of $D_{1}-D_{3}$.

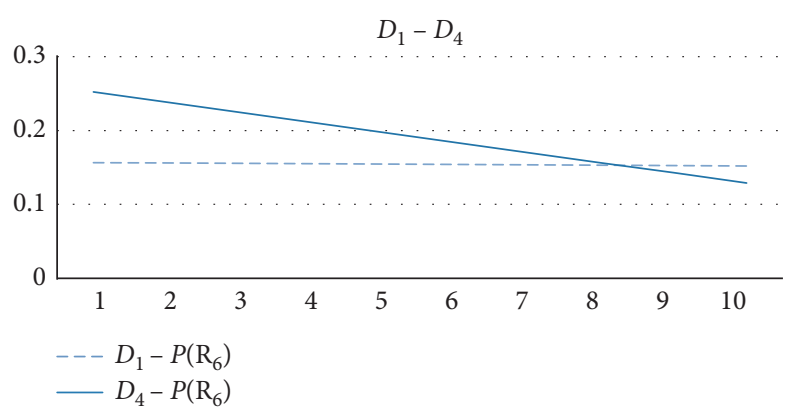

Figure 4: Sensitivity analysis of $D_{1}-D_{4}$.

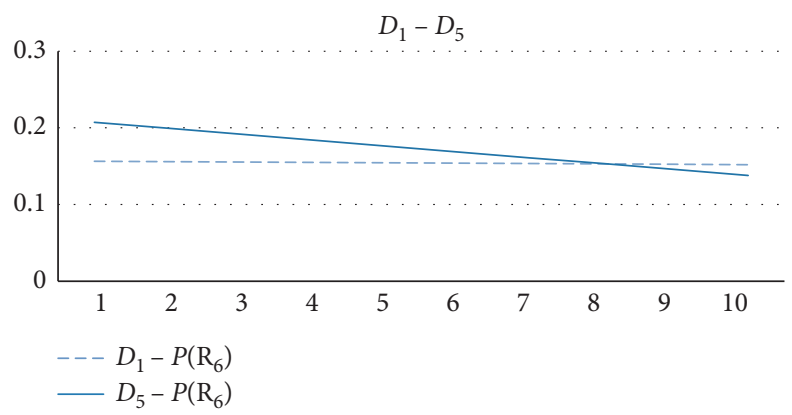

Figure 5: Sensitivity analysis of $D_{1}-D_{5}$. 


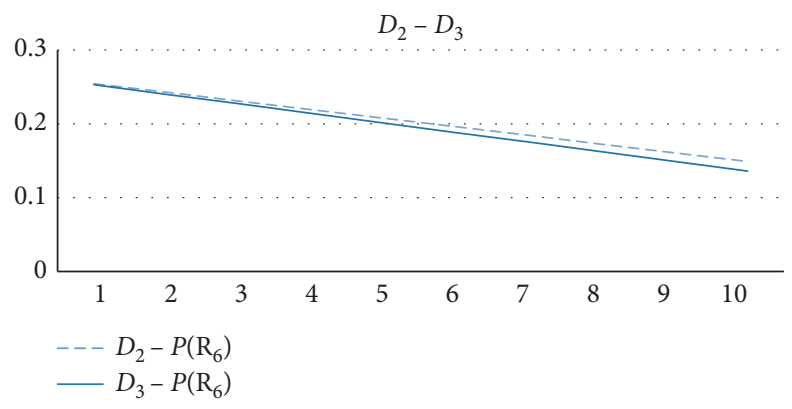

FIgURe 6: Sensitivity analysis of $D_{2}-D_{3}$.

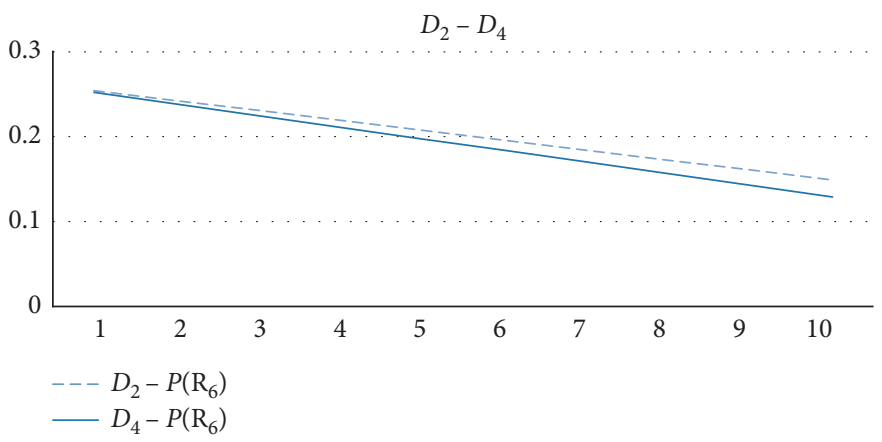

Figure 7: Sensitivity analysis of $D_{2}-D_{4}$.

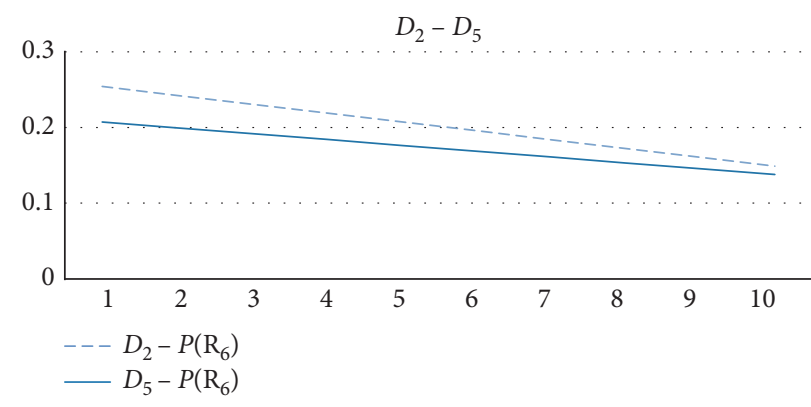

Figure 8: Sensitivity analysis of $D_{2}-D_{5}$.

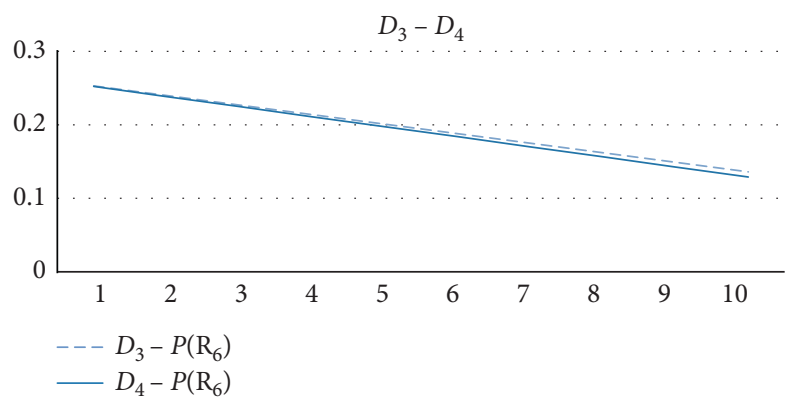

Figure 9: Sensitivity analysis of $D_{3}-D_{4}$. 


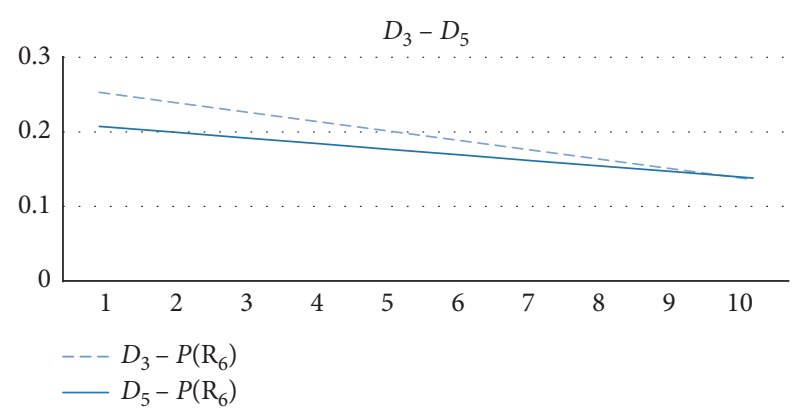

Figure 10: Sensitivity analysis of $D_{3}-D_{5}$.

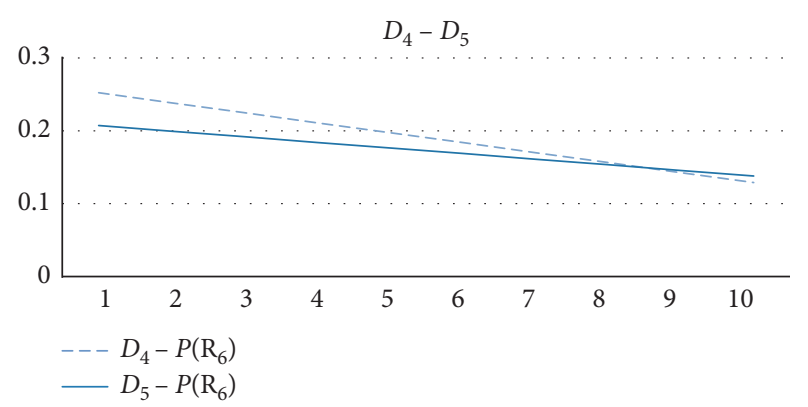

Figure 11: Sensitivity analysis of $D_{4}-D_{5}$.

bile triangle. In addition, the least efficient way to reduce the risk of death is to improve the success rate of trocar puncture surgery. These conclusions have certain guiding significance for the improvement of laparoscopic surgery of a hospital in Sichuan.

\section{Conclusion}

As a crucial factor of running smart city, risk flow management need to calculate accurately. Our BN_SC model modeled a special situation for risks flow in Bayesian net, which logical inference has to follow a strict chain. This is to describe surge process especially LC in this paper. The BN_SC computed the probabilities of risks in LC by Bayesian inference follow the particular process. Our model is a special Bayesian model for the specific system, limiting to the structure of the process, but can reduce computations in the suitable situations by the formula we presented.

The application of the BN_SC has proved its rationality and practicality. The method also has a way to combine the judgments and data of several hospitals to representative group judgments to deal with the weights of risks. Our model can be applied in other probabilities inference problems that have a strict chain to follow; for example, stock prices follow a timeline or story events follow a plotline.

\section{Data Availability}

The data used to support the findings of this study are available from the corresponding author upon request.

\section{Conflicts of Interest}

The authors declare that they have no conflicts of interest.

\section{Acknowledgments}

This work was partly supported by the National Natural Science Foundation of China (Grant no. 71861015), Distinguished Young Scholar Talent of Jiangxi Province (Grant no. 20192BCBL23008), the Humanities and Social Science Foundation of the Ministry of Education of China (Grant no. 18YJA630047), and the Humanities and Social Science Foundation of Universities of Jiangxi Province (Grant no. GL18122).

\section{References}

[1] A. Gelman and C. P. Robert, "“Not only defended but also applied": the perceived absurdity of bayesian inference," The American Statistician, vol. 67, no. 1, pp. 1-5, 2013.

[2] J. Yang, N. Xiong, A. V. Vasilakos et al., "A fingerprint recognition scheme based on assembling invariant moments for cloud computing communications," IEEE Systems Journal, vol. 5, no. 4, pp. 574-583, 2011.

[3] H. Zhenhua, X. Xin, Z. Honghao, and Z. MengChu, "An efficient group recommendation model with multiattentionbased neural networks," IEEE Transactions on Neural Networks and Learning Systems, vol. 31, pp. 1-14, 2020.

[4] R. Jiang, M. Shi, and W. Zhou, "A privacy security risk analysis method for medical big data in urban computing," IEEE Access, vol. 7, no. 12, pp. 143841-143854, 2019.

[5] J. Hu, Y. Sun, G. Li, G. Jiang, and B. Tao, "Probability analysis for grasp planning facing the field of medical robotics," Measurement, vol. 141, pp. 227-234, 2019.

[6] Q. Yue, L. Zhang, B. Yu, L. J. Zhang, and J. Zhang, “Two-sided matching for triangular intuitionistic fuzzy numbers in smart environmental protection," IEEE Access, vol. 7, pp. 4242642435, 2019.

[7] Q. Yue and L. Zhang, "Two-sided matching for hesitant fuzzy numbers in smart intelligent technique transfer," Mechanical Systems and Signal Processing, vol. 139, p. 106643, 2020.

[8] J. Sampaio, E. J. Drinkwater, and N. M. Leite, "Effects of season period, team quality, and playing time on basketball players' game-related statistics," European Journal of Sport Science, vol. 10, no. 2, pp. 141-149, 2010.

[9] A. C. Constantinou, N. E. Fenton, and M. Neil, "Profiting from an inefficient association football gambling market: prediction, risk and uncertainty using bayesian networks," Knowledge-Based Systems, vol. 50, no. 2, pp. 60-86, 2013.

[10] K. Tuyls, S. Maes, and B. Vanschoenwinkel, "Machine learning techniques for fraud detection," Master thesis, VUB, Brussels, Belgium, 2000.

[11] D. Koller, N. Friedman, and F. Bach, Probabilistic Graphical Models: Principles and Techniques, MIT press, Cambridge, MA, USA, 2009.

[12] N. Meinshausen and P. Bühlmann, "High-dimensional graphs and variable selection with the Lasso," The Annals of Statistics, vol. 34, no. 3, pp. 1436-1462, 2006.

[13] M. J. Wainwright and M. I. Jordan, "Graphical models, exponential families, and variational inference," Foundations and Trends ${ }^{\circledR}$ in Machine Learning, vol. 1, no. 1-2, pp. 1-305, 2008.

[14] Z. Ghahramani, "Learning dynamic bayesian networks," in International School on Neural Networks, Initiated by IIASS and EMFCSC, pp. 168-197, Springer, Berlin, Germany, 1997.

[15] D. Heckerman, D. Geiger, and D. M. Chickering, "Learning bayesian networks: the combination of knowledge and 
statistical data," Machine Learning, vol. 20, no. 3, pp. 197-243, 1995.

[16] R. E. Neapolitan, Learning Bayesian networks, Pearson Prentice Hall, Upper Saddle River, NJ, USA, 2004.

[17] S. A. Andersson, D. Madigan, and M. D. Perlman, "A characterization of markov equivalence classes for acyclic digraphs," The Annals of Statistics, vol. 25, no. 2, pp. 505-541, 1997.

[18] S. B. Gillispie and M. D. Perlman, "Enumerating markov equivalence classes of acyclic digraph dels," in Proceedings of the Seventeenth Conference on Uncertainty in Artificial Intelligence, Morgan Kaufmann Publishers Inc, Seattle, WA, USA, pp. 171-177, August 2001.

[19] J. Pearl, Causality: Models, Reasoning and Inference, Cambridge University Press, Cambridge, UK, 2000.

[20] R. Zinke, J. Melnychuk, F. Köhler, and U. Krause, "Quantitative risk assessment of emissions from external floating roof tanks during normal operation and in case of damages using Bayesian Networks," Reliability Engineering \& System Safety, vol. 197, p. 106826, 2020.

[21] A. Bhattacharjee, "Bayesian competing risks analysis without data stratification," Clinical Epidemiology and Global Health, vol. 8, no. 1, pp. 265-270, 2020.

[22] F. Sanchez, E. Bonjour, J.-P. Micaelli, and D. Monticolo, “An approach based on bayesian network for improving project management maturity: an application to reduce cost overrun risks in engineering projects," Computers in Industry, vol. 119, p. 103227, 2020.

[23] K. Masmoudi, L. Abid, and A. Masmoudi, "Credit risk modeling using Bayesian network with a latent variable," Expert Systems with Applications, vol. 127, no. 8, pp. 157-166, 2019.

[24] S. Punyamurthula and F. Badurdeen, "Assessing production line risk using bayesian Belief networks and system dynamics," Procedia Manufacturing, vol. 26, no. 8, pp. 76-86, 2018.

[25] J. H. Lee, S. Kim, I. Lee et al., "A risk prediction model for medical treatment failure in tubal pregnancy," European Journal of Obstetrics \& Gynecology and Reproductive Biology, vol. 225, pp. 148-154, 2018.

[26] S. Stonelake, P. Thomson, and N. Suggett, "Outcomes following emergency laparotomy: a comparison of predicted possum morbidity with the Clavien-Dindo classification of surgical complications," International Journal of Surgery, vol. 11 , no. 8 , p. $717,2013$. 